\title{
XXIX. On the conduction of electricity through gases between parallel plates
}

\author{
Alfred A. Robb
}

To cite this article: Alfred A. Robb (1905) XXIX. On the conduction of electricity through gases between parallel plates, Philosophical Magazine Series 6, 10:56, 237-242, DOI: 10.1080/14786440509463367

To link to this article: http://dx.doi.org/10.1080/14786440509463367

曲 Published online: 16 Apr 2009.

Submit your article to this journal $₫$

Џ Article views: 3

Q View related articles $\square$

Citing articles: 1 View citing articles 5 


\section{[ 237 ]}

XXIX. On the Conduction of Electricity through Gases between Parallel Plates. By Alfred A. RodB *

THE differential equation

$$
\begin{aligned}
& \frac{d^{2} \mathrm{X}^{2}}{d x^{2}}=8 \pi e\left(\frac{1}{\mathrm{R}_{1}}+\frac{1}{\mathrm{R}_{2}}\right)\left\{q-\frac{a}{e^{2} \mathrm{X}^{2}\left(\mathrm{R}_{1}+\mathrm{R}_{2}\right)^{2}}\right. \\
&\left.\times\left(i+\frac{\mathrm{R}_{2} d \mathrm{X}^{2}}{8 \pi d x}\right)\left(i-\frac{\mathrm{R}_{1} d \mathrm{X}^{2}}{8 \pi d x}\right)\right\}
\end{aligned}
$$

occurs in the theory of the conduction of electricity through gases between parallel plates.

In this equation $X$ represents the electric intensity at a point defined by the coordinate $x$, measured in a direction perpendicular to the plates. $i$ represents the current per unit area $; R_{1}$ and $R_{2}$ are the velocities of the positive and negative ions under unit electric intensity; $q$ is the rate of ionization per unit volume and $\alpha$ is the coefficient of recombination.

The equation has been solved by Professor J. J. Thomson for the case when $R_{1}=R_{2}$; but in general it does not lend itself to solution. It is proposed here to show (i.) that this equation may readily be transformed so that it becomes a characteristic one for any gas under definite conditions of temperature and pressure; and (ii.) that for any gas for which $R_{1}$ and $R_{2}$ are unequal there exist two pressures at which the equation becomes soluble.

In order to effect the first of these objects, we have only to write :

$$
\left.\begin{array}{l}
\mathrm{X}^{2}=\frac{i^{2}}{q} y^{2} \\
x=\frac{i}{q} v ;
\end{array}\right\} . . . . .
$$

and the equation becomes

$$
\begin{aligned}
\frac{d^{2} y^{2}}{d v^{2}}=8 \pi e\left(\frac{1}{\mathrm{R}_{1}}+\frac{1}{\mathrm{R}_{2}}\right) & \left\{1-\frac{\alpha}{e^{2} y^{2}\left(\mathrm{R}_{1}+\mathrm{R}_{2}\right)^{2}}\right. \\
& \left.\times\left(1+\frac{\mathrm{R}_{2} d y^{2}}{8 \pi d v}\right)\left(1-\frac{\mathrm{R}_{1} d y^{2}}{8 \pi d v}\right)\right\} .
\end{aligned}
$$

The form of this equation does not depend either on the strength of the current or the intensity of the ionization, and therefore is characteristic for the gas.

In order to show that for certain pressures this equation

- Communicated by the Author. 
takes a soluble form, we shall write it

$$
\frac{d^{2} y^{2}}{d v^{2}}=a+\frac{b}{y^{2}}+\frac{c d y^{2}}{y^{2} d \bar{d}}+\frac{\epsilon}{2 y^{2}}\left(\frac{d y^{2}}{d v}\right)^{2}
$$

where

$$
\begin{aligned}
& x=\frac{8 \pi e\left(\mathrm{R}_{1}+\mathrm{R}_{2}\right)}{\mathrm{R}_{1} \mathbf{R}_{2}}, \\
& b=-\frac{8 \pi \alpha}{e \mathrm{R}_{1} \mathbf{R}_{2}\left(\mathrm{R}_{1}+\mathrm{R}_{2}\right)}, \\
& c=-\frac{\alpha\left(\mathrm{R}_{2}-\mathrm{R}_{1}\right)}{e \overline{\mathrm{R}_{1} \mathbf{R}_{2}\left(\mathrm{R}_{1}+\mathrm{R}_{2}\right)},} \\
& \epsilon=\frac{\alpha}{4 \pi e\left(\mathrm{R}_{1}+\overline{\mathrm{R}_{2}}\right)} .
\end{aligned}
$$

It is to be observed here that $\epsilon$ is the same quantity as that introduced by Langevin (Recherches sur les $G a z$ ionisés, University of Paris, 1902), and denoted by the same letter.

It follows from the physical meaning which he gives it that $\epsilon$ must be a positive fraction.

In order to reduce equation (4) we make the following series of substitutions: 1 st. Putting $\frac{d y^{2}}{d v}=-\frac{2}{\epsilon} \frac{w}{z}$ and $y^{\epsilon}=\frac{1}{z}$, we get

$$
w \frac{d w}{d z}-c w+\frac{l \epsilon}{2} z=-\frac{a \epsilon}{z} z^{1-\frac{2}{\epsilon}} . . . .
$$

2nd. Putting $w=\frac{s}{\mathrm{~K}} \frac{d z}{d s}$, where $\mathrm{K}$ is a constant to be afterwards determined, we get

$$
s^{2} \frac{d^{2} z}{d s^{2}}+(1-c \mathrm{~K}) s \frac{d z}{d s}+\frac{b \epsilon}{2} \mathrm{~K}^{2} z=-\frac{a \epsilon}{2} \mathrm{~K}^{2} z^{1-\frac{2}{\epsilon}} . .
$$

3rd. Putting $\boldsymbol{\theta}=\approx s^{n}$, we have

$$
\frac{1}{s^{n-2}} \frac{d^{2} \theta}{d s^{2}}=s^{2} \frac{d^{2} z}{d s^{2}}+z n s \frac{d z}{d s}+n(n-1) \hat{n} .
$$

If, therefore, $\mathrm{K}$ and $n$ be determined by the equations

$$
1-c \mathrm{~K}=2 n \text {, }
$$

and

$$
\left.\frac{b \epsilon}{2} K^{2}=n(\urcorner-1\right) ;
$$


we have $n$ given by the equation

$$
\frac{b \epsilon}{2 c^{2}}(1-2 n)^{2}=n(n-1) ; . . . \quad .
$$

and the differential equation becomes

$$
\frac{d^{2} \theta}{d s^{2}}=-\frac{a}{b} n(n-1) \theta^{1-\frac{2}{\epsilon} s^{\frac{2 n}{\epsilon}-2} . ~ . ~ . ~ . ~}
$$

Now equation (7) has two roots. If $n_{1}$ and $n_{2}$ be these two values, then $n_{1}+n_{2}=1$; and so unless $n_{1}=n_{2}=\frac{1}{2}$ there will be two equations of form (8) corresponding to any one of form (4). Regarded merely as a differential equation, (8) may be solved in several cases :-

$$
\begin{aligned}
& \text { (i.) When } a=0 . \\
& \text { (ii.) When } \epsilon=2 . \\
& \text { (iii) When } n=\frac{1}{2} . \\
& \text { (iv.) When } n=\epsilon \text { or } 1-\epsilon \text {. }
\end{aligned}
$$

As regards case (i.), our problem does not allow of $a$ being zero.

Further, $\epsilon$ is necessarily a positive fraction, and so case (ii.) is useless for our purpose.

Case (iii.) corresponds to $c=0$, or in other words to $\mathbf{R}_{1}=\mathbf{R}_{2}$, and the equation of conduction under these circumstances has already been solved by Professor J. J. Thomson ('Conduction of Electricity through Gases,' p. 66, or Phil. Mag. vol, xlvii. p. 253, 1899).

Case (iv.) requires

$$
\frac{b \epsilon}{2 c^{2}}=\frac{\epsilon(\epsilon-1)}{(i-2 \epsilon)^{2}} \text { or } \quad b=\frac{2(\epsilon-1)}{(1-2 \epsilon)^{2}} c^{2}
$$

But

$$
\frac{l \epsilon}{2 c^{2}}=-\frac{\mathrm{R}_{1} \mathrm{R}_{2}}{\left(\mathrm{R}_{2}-\mathrm{R}_{1}\right)^{2}} \text {. }
$$

Thus we get either

$$
\text { or } \left.\begin{array}{rl}
\epsilon & =\frac{\mathrm{R}_{1}}{\mathrm{R}_{1}+\mathrm{R}_{2}} \\
\epsilon & =\frac{\mathrm{R}_{2}}{\mathrm{R}_{\mathrm{i}}+\mathrm{R}_{2}}
\end{array}\right\} \quad . . . .
$$

Now both these values of $\epsilon$ are positive fractions, and therefore are possible. For air $\frac{\mathrm{R}_{2}}{\mathrm{R}_{1}}=1.22$ approximately, 
and thus the values of $\frac{R_{1}}{R_{1}+R_{2}}$ and $\frac{R_{2}}{R_{1}+R_{2}}$ are respectively

Now Langevin gives $\epsilon=\cdot 27$ at $760 \mathrm{~mm}$. pressure and $\cdot 62$ at $1550 \mathrm{~mm}$. for the case of air.

Thus $\epsilon=.45$ corresponds to about 1.53 atmospheres, and $\epsilon=.55$ to about $1: 83$ atmospheres.

Accordingly, for air at these two pressures the equation has the desired form, and may be solved.

Taking, then, for simplicity $n=\epsilon(n=1-\epsilon$ gives of course the same final result), we hare

$$
\frac{d^{2} \theta}{d s^{2}}=-{ }_{0}^{a} \epsilon(\epsilon-1) \theta^{1-{ }_{\epsilon}^{2}}
$$

This gives

$$
\int \frac{n^{\prime} \theta}{\sqrt{\mathrm{C}_{1}-\frac{a \epsilon^{2}}{b} \theta^{2-{ }^{2}}}}+\mathrm{C}_{2}=s
$$

where $C_{1}$ and $C_{2}$ are constants of integration.

By reversing the various steps indicated, we may finally obtain $y$ and $v$ in terms of $\theta$ as an auxiliary variable; but it is desirable to introduce a slight change of notation in order that the final result may not contain a third and superfluous constant.

We accordingly write

and

$$
\phi\left(\frac{b C_{1}}{a \epsilon^{2}}\right)^{\frac{\epsilon}{2 \epsilon-2}}=\theta
$$

$$
\frac{\mathrm{C}_{2} \sqrt{-\overline{\mathrm{C}_{1}}}}{\left(\frac{b \mathrm{C}_{1}}{a \epsilon^{2}}\right)^{\frac{\epsilon}{2 \epsilon-2}}}=k_{1}
$$

a transformation which is only possible if $C_{1}$ be different from zero, and finally obtain $y$ and $v$ in terms of the auxiliary variable $\phi$. Thus

$$
\left.\begin{array}{l}
y=\frac{-c}{\epsilon(1-2 \epsilon)} \sqrt{\frac{2(1-\epsilon)}{a}\left\{\int \frac{d \phi}{\sqrt{\phi^{2}-\frac{2}{\epsilon}-1}}+k_{1}\right\}} \frac{1}{\phi_{\epsilon}^{-}} \\
v=\frac{2 c(1-\epsilon)}{c \epsilon^{2}(1-2 \epsilon)}\left\{\int \frac{\left.d \int \frac{d \phi}{\sqrt{\phi^{2-\frac{2}{\epsilon}-1}}+k_{1}}\right\} d \phi}{\left.\phi_{\epsilon}^{\frac{2}{2}} \sqrt{\phi^{2-\frac{2}{\epsilon}-1}}+\text { const. }\right\}}\right\}
\end{array}\right\}
$$

As may readily be proved by differentiation. 
Besides this solution, there is another corresponding to the case where $\mathrm{C}_{1}$ is zero, and when therefore the transformation from $\theta$ to $\phi$ fails.

In this case the relation between $y$ and $v$ can be expressed directly without the use of an auxiliary variable, and may readily be shown to be

$\pm 2 \sqrt{\frac{1-\epsilon}{2 a}} y-\frac{2 c}{a} \frac{1-\epsilon}{1-2 \epsilon} \log \left\{\frac{2 c}{1-2 \epsilon} \pm \sqrt{\frac{2 a}{1-\epsilon}} y\right\}=v+$ const.

The solution (11) may be somewhat simplified in form, for if we put

$$
\phi^{1-\frac{1}{c}}=\cosh \omega,
$$

and substitute the values of $c$ and $a$, we get

$$
\left.\begin{array}{l}
y=\frac{4 \pi}{\sqrt{\alpha}}\left\{\frac{-\epsilon}{1-\epsilon} \int \frac{i \omega}{\cosh ^{\frac{1}{1-\epsilon}} \omega}+k_{1}\right\} \cosh ^{\frac{1}{1-\epsilon}} \omega \\
v=\frac{\mathrm{R}_{2}-\mathrm{R}_{1}}{\sqrt{ } \alpha} \frac{\epsilon}{1-2 \epsilon}\left\{\int y d \omega+k_{2}\right\},
\end{array}\right\} .
$$

where $\sqrt{ } \alpha$ is to be employed with the same sign in both places and where

$$
\text { or } \quad \begin{aligned}
\epsilon & =\frac{R_{1}}{R_{1}+R_{2}}, \\
& \epsilon=\frac{R_{3}}{R_{1}+R_{2}},
\end{aligned}
$$

according as the pressure is such as to make

$$
\begin{aligned}
& \alpha=4 \pi e \mathrm{R}_{1}, \\
& \text { or } \quad \alpha=4 \pi e \mathbf{R}_{2} \text {. }
\end{aligned}
$$

The integrals in (13) may be evaluated in finite form when $\frac{1}{1-\epsilon}$ is an integer, and when therefore the velocity of one ion is an exact multiple of the velocity of the other. In other cases they may be easily calculated*.

The other solution (12) may be put in the form

$$
\begin{aligned}
& 4 \pi \epsilon^{2}{ }_{1-\epsilon \mathrm{R}_{2}-\mathrm{R}_{1}}^{1-2 \epsilon} \log \{\left.-\frac{8 \pi \epsilon}{1-2 \epsilon}\left(\frac{1}{\mathrm{R}_{1}}-\frac{1}{\mathrm{R}_{2}}\right) \pm 2 \sqrt{\frac{\alpha}{\epsilon(1-\epsilon) \mathrm{R}_{1} \mathrm{R}_{2}}} y\right\} \\
& \pm 2 \sqrt{\frac{\epsilon(1-\epsilon) \mathrm{R}_{1} \mathrm{R}_{2}}{\alpha}} y=v+\text { const. . (14) }
\end{aligned}
$$

* The author has calculated a table of these integrals for $\epsilon=\frac{4}{q}$, which is approximately one of the values for the case of air. This he hopes to give in a supplementary paper at an early date.

Plil. Mag. S. 6. Vol. 10. No. 56. Aug. 1905. 
in which, as before, $\epsilon$ has the values $\frac{R_{1}}{R_{1}+R_{2}}$ and $\frac{R_{2}}{R_{1}+R_{2}}$, corresponding respectively to the pressures at which

$$
\alpha=4 \pi e \mathrm{R}_{1} \text { and } \alpha=4 \pi e \mathrm{R}_{2} .
$$

Note.-The idea of adjusting the pressure so as to render the equation of conduction of electricity between parallel plates more manageable occurred independently to Mr. G. W. Walker and myself. It was used by Mr. Walker to complete the integration in the case when $R_{1}=R_{2}$. It was shown by him (Phil. Mag. Nov. 1904) that this may be done if $\frac{x}{8 \pi e \mathrm{R}}=2, \frac{1}{2}, \frac{1}{3}, \frac{1}{4}$, and he selected 2 as the simplest.

I pointed out to him in a letter that according to Langevin's theory, $\epsilon=\frac{\alpha}{8 \pi e \mathrm{~h}}$ must be a fraction, and that therefore the value 2 was physically impossible.

He replies that he had not observed the limitation imposed by Langevin's theory, and suggests that I should lay emphasis on the point that while the value $\frac{\alpha}{8 \pi e \mathrm{R}}=2$ is possible if $a$ be constant and $\mathbf{R}$ vary inversely as the pressure according to McClung's and Rutherford's results, yet according to Langevin $\frac{\alpha}{8 \pi e \overline{\mathrm{R}}}$ can never be greater than unity.

There is thus a serious difference to be explained.

XXX. The Rate of Recombination of Ions in Gases. By O. W. Richardoson *.

TTHE following is an attempt to explain the variation of the rate of recombination of ions formed in gases with the pressure of the gas. The basis of the method is a theorem due to Langevin $\dagger$, which may be stated as follows :- If we describe around each negative ion a sphere whose radius is arbitrary, except that it must be large compared with the mean free path of an ion and small compared with the average distance between two ions--both of which conditions are capable of being satisfied in the case of ionization produced by Röntgen rays and radium radiation, in gases at moderate pressures - then the number of positive ions which

* Communicated by the Author.

† Thèses de l'Université de Paris, A. 431, p. 103 (Gauthier Villars). 